\title{
Robust Epileptic Seizure Detection on Wearable Systems with Reduced False-Alarm Rate
}

\author{
Renato Zanetti, Amir Aminifar, and David Atienza
}

\begin{abstract}
Epilepsy affects more than 50 million people and ranks among the most common neurological diseases worldwide. Despite advances in treatment, one-third of patients still suffer from refractory epilepsy. Wearable devices for real-time patient monitoring can potentially improve the quality of life for such patients and reduce the mortality rate due to seizure-related accidents and sudden death in epilepsy. However, the majority of employed seizure detection techniques and devices suffer from unacceptable false-alarm rate. In this paper, we propose a robust seizure detection methodology for a wearable platform and validate it on the Physionet.org CHB-MIT Scalp EEG database. It reaches sensitivity of 0.966 and specificity of 0.925 , and reducing the false-alarm rate by $34.7 \%$. We also evaluate the battery lifetime of the wearable system including our proposed methodology and demonstrate the feasibility of using it in real time for up to 40.87 hours on a single battery charge.
\end{abstract}

Clinical relevance - We propose a methodology to increase classification robustness and reduce the false-alarm rate for epileptic seizure detection using wearable systems.

\section{INTRODUCTION}

The World Health Organization (WHO) estimates more than 50 million people suffering from epilepsy worldwide [1]. Despite the advances in treatment, approximately threequarters of patients do not have access to drugs [1] and around one-third of the cases are drug-resistant (cases classified as refractory epilepsy) [2]. Previous studies have demonstrated that most of the patients with refractory epilepsy would use a device for long-term epileptic-seizure monitoring [3], [4]. Although such device have the potential of improving quality of life of people with epilepsy (PWE), the surveys report concerns with social stigma and the possibility of PWE being exposed by wearing such technology. Moreover, PWE also demonstrate a strong preference for removable devices resembling usual daily-life accessories.

Most of the existing devices for epileptic-seizure tracking are watch like, using peripheral measured signals to assess patient status. In particular, (1) SmartWatch Inspyre ${ }^{T M}$ by Smart Monitors uses an accelerometer (ACC) to monitor abnormal and rhythmic movements during seizures, reaching sensitivity of 92\% [5]; (2) Embrace and E4 wristbands (Empatica Inc.) feature an electrodermal activity (EDA) sensor extra to an ACC, reaching sensitivity between $92 \%$ and $100 \%$ [5]; (3) Nightwatch, an armband device, also uses an ACC

\footnotetext{
*This work has been partially supported by the e-Glass project (EPFL Enable project No. 6.1828), the MyPreHealth Cyber-Human project (Hasler Foundation project No. 16073), the WiTNESS Promobilia Foundation project (Ref. 18079), and Sklodowska-Curie GA No. 754354.

R. Zanetti, A. Aminifar, and D. Atienza are with the Embedded Systems Laboratory (ESL) of the Swiss Federal Institute of Technology (EPFL), 1015 Lausanne, Switzerland \{renato.zanetti, amir.aminifar, david.atienza\}@epfl.ch
}

sensor but, in this case, combined with photoplethysmography (PPG). The latter presented $86 \%$ of mean sensitivity on a study with more than one type of night seizures [6]. Although presenting high sensitivity values, these devices operation focus on the detection of one type of seizure, characterized by violent body movements, the generalized tonic-clonic seizures (GTCS).

In spite of advances in analysis and techniques for epileptic seizure assessment, electroencephalography (EEG) is still an essential tool in the diagnosis and monitoring of PWE [7]. Video-EEG is the current clinical gold standard in epileptic seizures diagnosis. However, it is a demanding procedure typically performed at hospital facilities, while the patient is kept under camera surveillance as long as the EEG acquisition lasts (up to 1-week). Even though there are several wireless EEG acquisition systems, existing commercial equipment are non-portable and/or rely on EEGcaps.

Specialized wearable technologies can provide a suitable solution for mobility constraints and stigma associated with EEG-systems, enabling long-term and accurate epileptic seizure monitoring based on biosignals, meanwhile hidden in inconspicuous packaging. Nevertheless, the use of resource-constrained platforms in outpatient monitoring is a challenging problem [8]. The operation in real-world is error-prone and requires considerable processing power and extended battery lifetime. Although there are various studies addressing automatic seizure detection based on EEG and machine learning [9], [10], [11], [12], to the best of our knowledge, there is no commercial wearable solution on the market using EEG to monitor epilepsy patients in outpatient conditions.

In order to build such a device, the authors of [10] assessed the possibility of using only two bipolar channels of EEG over the temporal and frontal lobes in comparison with using data from full cap. The reduced set of electrodes embodied on a glasses form factor produces a technological concept capable of tackling both social stigma and wearability problems. In this work, we propose and validate a robust methodology for epileptic seizure monitoring, based on a previous wearable system [10], to reduce false-alarm rate in real-time epileptic seizure detection. The main contributions of this work are:

1. We propose a robust epileptic seizure detection methodology based on data fusion, combining multiple data to improve classification model. We validate the proposed methodology on the CHB-MIT database [13] and improve the FAR by $34.70 \%$, compared to the previous study [10].

2. We implement the proposed methodology on an state- 


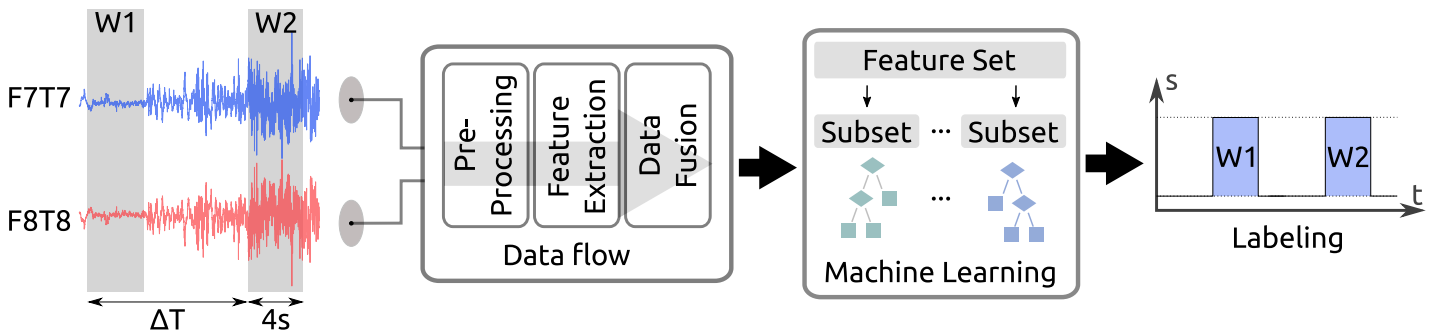

Fig. 1. Proposed methodology for seizure detection.

of-the-art wearable platform, and assess its battery lifetime. Our results show that it can reach up to 40.87 hours of continuous monitoring on a single battery charge, which enables a full day of work with a reduced amount of false-alarms.

\section{Robust Epileptic Seizure Detection Approach}

We propose a seizure detection methodology based on the edge computing paradigm, thus processing all the available data on the device. The main processing pipeline is divided into four blocks (Fig. 1): signal pre-processing, feature extraction, data fusion, and classification based on a lightweight machine-learning algorithm. These blocks are further described in the following subsections.

\section{A. EEG Acquisition and Pre-Processing}

As presented in [10], we use only two bipolar channels of EEG (F7T7 and F8T8, international 10-20 system [14]). We use an EEG front-end for signal conditioning and a reduced setup to obtain a minimalist wearable platform for seizure detection (further described in Section III-B). In terms of signal pre-processing, we filter the EEG signal using a bandpass filter (Butterworth, $4^{\text {th }}$ order) with cutoff frequencies of 0.3 and $45 \mathrm{~Hz}$. Moreover, we apply a discrete wavelet transform (DWT) to decompose the EEG signal before extracting nonlinear features to improve the classification accuracy [15].

\section{B. Feature Extraction}

We extract a total of 54 features from each four-second EEG epoch, namely:

1) bandpower: we estimate the power spectral density (PSD) by using periodogram method [16]. The PSD estimate is used to calculate the bandpower in the following frequency bands: delta $[0.5,4] \mathrm{Hz}$, theta $[4,8] \mathrm{Hz}$, alpha $[8,12] \mathrm{Hz}$, beta $[13,30] \mathrm{Hz}$, and gamma [30, 45] Hz. Additionally, we calculate the total power and power for $[0,0.1] \mathrm{Hz},[0.1$, $0.5] \mathrm{Hz}$, and [12, 13] Hz bands. For each of the eight bands, we also calculate the relative power to the total power. In total, we obtain 17 power features from one EEG epoch.

2) entropy: each EEG epoch is decomposed in approximated (A) and detailed (D) coefficients down to level seven using a Daubechies 4 (DB4) basis function. We extract sample entropy [17] from detail coefficients at level six (D6) and seven (D7). In addition, we extract permutation entropy [18], Renvi, Shannon and Tsallis entropy [19], from detail coefficients D3, D4, D5, D6, and D7.

\section{Data Fusion}

Data fusion is a technique to bind information from multiple sources to obtain more consistent, informative or comprehensive, and accurate representation about a process or entity [20]. In particular, data fusion can be used to improve epileptic seizure detection robustness to false-positives by increasing data variability. For instance, the period in between the onset and offset of an annotated seizure may have one or more seizure bursts, thus containing periods of less synchronous epileptic activity. Considering that all available data of this seizure is used for training a suitable model to identify an ictal EEG epoch, such less synchronous part of signal may increase the generalization error of the model. To tackle this problem, we propose to use an approach based on data fusion.

Our data fusion approach combines two epochs of EEG to form a new set of features representing different moments of a seizure. For each chosen EEG epoch for the previous work methodology [10], we add a second epoch fusing it as double set of features. These second set is taken from a randomly selected EEG epoch, picked before the first EEG epoch but with a separation time $(\Delta \mathrm{T})$ limited to one-fourth of the average seizure time per subject (respecting ictal and inter-ictal periods). This way we can reduce the probability of having two epochs of less synchronous epileptic activities in the double set of features and generate a more robust model to false positives.

\section{Seizure Classification}

In this paper, we choose Random Forest (RF) as the classification algorithm as it is lightweight, suitable for resourceconstrained platforms, which has already been shown to provide promising results for epilepsy detection [21]. The the strength of individual trees and their diversity contributes to the performance of a RF ensemble of classification and regression trees. According to Breiman [22] proposed method, each tree of a random forest ensemble is grown independently on a bootstrap sample of the training data, a method called bagging. In consequence, a RF-based approach provides major improvement in the classification accuracy compared to decision trees, while mitigating the influence of overfitting.

\section{EXPERIMENTAL SETUP}

In this section, we present the considered epilepsy data, our target wearable platform, and power measurement settings used during performance evaluation of our proposed methodology. 


\section{A. Epilepsy Database}

We use the CHB-MIT Scalp database [13] to evaluate the performance of seizure detection. It contains data of 23 subjects ( 5 males and 17 females, $9.9 \pm 5.6$ years old), sampled at $256 \mathrm{~Hz}$ with 16-bit resolution according to the international 10-20 system (bipolar montage). There are 182 annotated seizures and data is available in a total of 664 files. In particular, subjects S6 and S16 present only relatively short seizures $(15.30 \mathrm{~s} \pm 2.87 \mathrm{~s}$ and $8.40 \mathrm{~s} \pm 2.27 \mathrm{~s}$, respectively) as, on average, a seizure is expected to last, typically, more than a minute [23]. Therefore, due to the limited ictal samples, results of these patients were not taken into account for FAR calculation.

\section{B. Implementation Platform}

We considered the platform described in [10], but including a hardware update, using validated off-the-shelf components, for medical applications. Our platform is based on the: (1) STM32L476 ARM cortex-M4 microcontroller $(\mu \mathrm{C})$, running at up to $80 \mathrm{MHz}$, including $128 \mathrm{kB}$ of RAM memory and featuring a floating-point unit to speed-up signal processing; (2) ADS1299 EEG front-end, a complete EEG system-on-chip in reduced package size; (3) BlueNRG-MS, Bluetooth low energy (BLE) network processor. Finally, we also have an ultra-low power triaxial accelerometer as another sensor that could be included on the seizure detection methodology. In terms of firmware, our application is based on the FreeRTOS, a real-time operating system kernel for embedded devices.

\section{Power Measurements}

To account for a realistic power consumption value, we measure the current consumption and determine the application duty cycle (i.e. percentage of processing time over an execution period) to run the feature extraction and model inference steps of both epileptic seizure detection methodology (our proposed and the previous work methodologies [10]). For this procedure, we use our platform running at maximum clock $(80 \mathrm{MHz})$ and consider to have an inference of the subject condition each four seconds. We use a shunt resistor and circuit based on the AD625 amplifier for current measurement with a TDS2024 oscilloscope.

\section{Performance Metrics}

We consider as classification performance metrics the sensitivity (sens), specificity (spec), and their geometric mean (GMEAN), which are defined as follows:

$$
\begin{gathered}
S E N S=\frac{t p}{t p+f n}, \quad S P E C=\frac{t n}{t n+f p} \\
G M E A N=\sqrt{S E N S \cdot S P E C} .
\end{gathered}
$$

We also consider the false-discovery rate (FDR) and falsealarm rate (FAR) per hour, which are defined as follows:

$$
F D R=\frac{f p}{t p+f p}, \quad F A R=3600 \cdot F D R .
$$

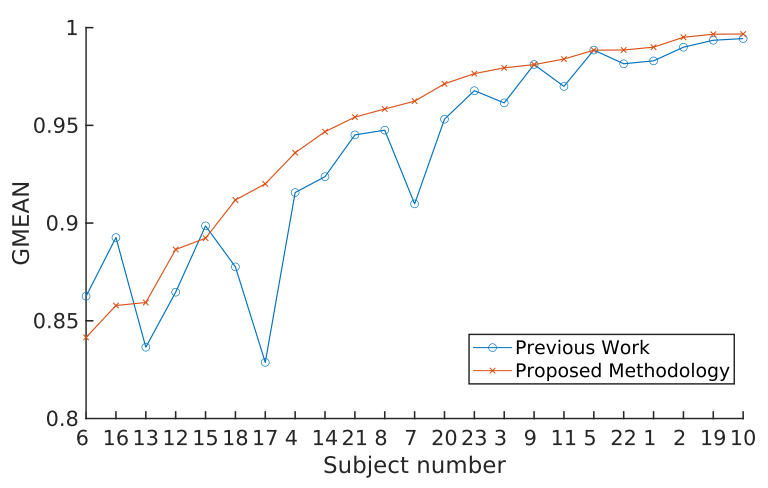

Fig. 2. Detection quality (GMEAN) for our proposed methodology (red) and previous work (blue).

\section{E. Performance Assessment}

To evaluate our proposed methodology, we use a personalized setting for each subject in the database, where we divide data according to the ictal and inter-ictal phases. The inter-ictal period in the CHB-MIT Epilepsy database is approximately 300 times longer than the ictal (summing up all seizure and available data time). For a realistic assessment of the FAR, the number of inter-ictal epochs used is approximately 300 times the ictal (only for testing).

We apply our feature extraction and data fusion approach per each data file. Each ictal period data is treated as an unit for training or testing. The same occurs per files without seizures. We only pick data from the inner half of the file with no seizure, avoiding proximity to neighboring seizures. Moreover, all available files per subject are used during feature extraction. Finally, the data is divided into training (70\%) and testing (30\%) sets and we use various combinations of data units during the split for a 10-fold cross-validation.

\section{EXPERIMENTAL RESULTS}

In this section we present the performance of our proposed methodology in terms of classification sensitivity, specificity, and false-alarm rate. Moreover, we also present the battery lifetime assessment.

\section{A. Seizure Detection Performance}

Fig. 2 presents the GMEAN per subject (vertical axis) for our proposed methodology and for the previous work [10]. We obtained a GMEAN across all subjects of 0.945 (SENS of 0.966 and SPEC of 0.925) using our proposed methodology, slightly higher than the GMEAN of 0.932 (SENS of 0.958 and SPEC of 0.908) when using the previous work approach. Moreover, we can observe a generalized increment in GMEAN for most of the subjects when using our proposed methodology, which can be seen already as an indication of the reduction in the false-positive rate.

Overall, the average FAR dropped from 1.078 to 0.704 (34.7\%) per hour, when applying our methodology in comparison with the previous work's. As it can be seen in Fig. 3, in the best case (Subject 19), our proposed methodology is able to remove all false positives. In fact the use of our proposed methodology represents a FAR improvement higher than $20 \%$ for 15 out of 23 subjects. However, in our worst-case scenario, Subject 5 presents a $12.17 \%$ increment 


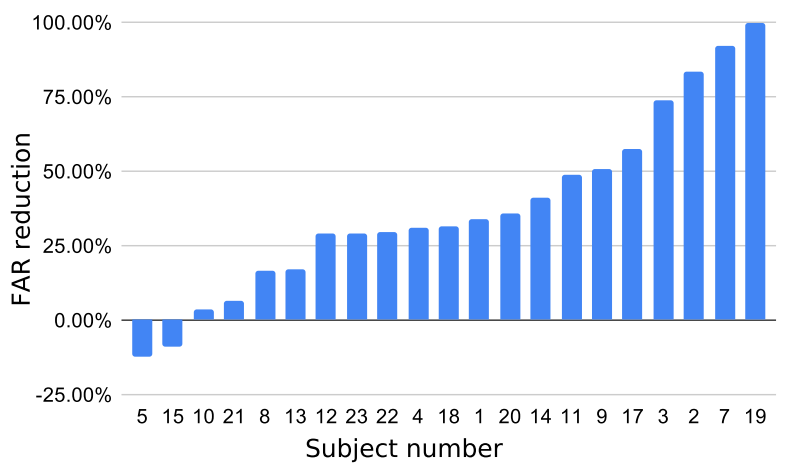

Fig. 3. FAR improvement among all subjects when using our proposed methodology vs previous work.

in FAR. This increment in FAR could be explained by an increment in false positives due to overfitting.

\section{B. Battery Lifetime}

Running both methodologies on our platform, we measure a system current consumption of approximately $18.75 \mathrm{~mA}$ with the $\mu \mathrm{C}$ in run mode and $6.55 \mathrm{~mA}$ in sleep mode. Table I shows execution duty cycle (four-second window), average current consumption and battery lifetime for both approaches. We activate the sleep mode when $\mu \mathrm{C}$ is idle for calculations (keeping basic operations as data acquisition related in interrupt service routines, which introduces about $5 \%$ processing overhead), thus we can reduce system average current consumption. Therefore, by running the processing approach at maximum speed, which lasts approximately $27.9 \mathrm{~ms}$ per EEG epoch for both channels, it is possible to reduce the duty cycle and the total power consumption. Considering a battery of $300 \mathrm{mAh}$, our data fusion proposed approach could be executed on the wearable platform for up to 40.87 hours of continuous monitoring on a single battery charge. The use of our proposed methodology slightly increases the energy consumption of the device if compared to the battery lifetime (41.37 hours) achieved employing the methodology in the previous work but increases FAR by $34.7 \%$. However, both approachs achieve more than a day of operation, which is considered satisfactory for the majority of patients surveyed in [4].

TABLE I

ESTIMATED BATTERY LIFETIME.

\begin{tabular}{cccc}
\hline Methodology & D. Cycle (\%) & Current (mA) & Lifetime (h) \\
\hline Previous work [10] & 5.76 & 7.25 & 41.37 \\
Our methodology & 6.52 & 7.34 & 40.87 \\
\hline
\end{tabular}

\section{CONCLUSION}

Wearable devices can enable long-term monitoring of epileptic seizures. However, as important as the classification accuracy, a tracking device needs to be robust to falsealarm occurrences when used in everyday life. This work has presented a new methodology to improve the robustness of a wearable device in terms of FAR. Moreover, we have introduced a novel data fusion phase to improve robustness to false positives by picking a second EEG epoch over the ictal or inter-ictal periods, adding data variability. Our results show that the proposed methodology reaches $94,5 \%$ of geometric mean between sensitivity and specificity for all original subjects of the CHB-MIT Epilepsy database, while reducing FAR by $34.7 \%$. All in all, this methodology can be used in real-time wearables, thus allowing up to 40.87 hours of continuous monitoring on a single battery charge.

\section{REFERENCES}

[1] World Health Organization, Epilepsy: a public health imperative, Accessed on Jan 27th 2020, [Online], Available on: https://go.epfl.ch/Who2019Epilepsy, 2019.

[2] A. Ulate-Campos et al., Automated seizure detection systems and their effectiveness for each type of seizure, Seizure, vol. 40, pp. 88-101, Aug. 2016

[3] C. Hoppe et al., Novel techniques for automated seizure registration: patients' wants and needs, Epilepsy \& Behavior, vol. 52, pp. 1-7, 2015.

[4] E. Bruno et al., Wearable technology in epilepsy: The views of patients, caregivers, and healthcare professionals, Epilepsy \& Behavior, vol. 85, pp. 141-149, Aug 2018.

[5] G. Regalia et al., Multimodal wrist-worn devices for seizure detection and advancing research: Focus on the Empatica wristbands, Epilepsy Research, vol. 153, pp. 79-82, 2019.

[6] J. Arends et al., Multimodal nocturnal seizure detection in a residential care setting, Neurology, vol. 91, no. 21, pp. 2010-2019, Nov. 2018.

[7] W. O. Tatum et al., Clinical utility of EEG in diagnosing and monitoring epilepsy in adults. Clinical Neurophysiology, vol. 129, no. 5, pp. 1056-1082, 2018.

[8] R. Braojos et al., Ultra-low power design of wearable cardiac monitoring systems, in 2014 51st ACM/EDAC/IEEE Design Automation Conference (DAC), 2014, pp. 1-6.

[9] Y. Park et al., Seizure prediction with spectral power of EEG using cost-sensitive support vector machines, Epilepsia, vol. 52, no. 10, pp. 1761-1770, Oct. 2011

[10] D. Sopic, A. Aminifar, and D. Atienza, e-Glass: A Wearable System for Real-Time Detection of Epileptic Seizures, in 2018 IEEE International Symposium on Circuits and Systems (ISCAS), Florence,Italy, 2018, pp. 1-5.

[11] D. P. Ortiz; A. Aminifar; D. Atienza, A Self-Learning Methodology for Epileptic Seizure Detection with Minimally Supervised Edge Labeling, in Design, Automation and Test in Europe (DATE), Florence, Italy, Mar. 2019, pp. 764 - 769.

[12] A. Thomas, A. Aminifar, D. Atienza, Noise-Resilient and Interpretable Epileptic Seizure Detection, in 2020 IEEE International Symposium on Circuits and Systems (ISCAS), Sevilha, Spain, May 2020, unpublished.

[13] A. Shoeb, Application of Machine Learning to Epileptic Seizure Onset Detection and Treatment, Ph.D. Dissertation, Massachusetts Inst. of Technology, MA, 2009.

[14] G. H. Klem et al., The ten-twenty electrode system of the International Federation. The International Federation of Clinical Neurophysiology, Electroencephalography and Clinical Neurophysiology (Supplement), vol. 52, pp. 3-6, 1999.

[15] H. Ocak, Automatic detection of epileptic seizures in EEG using discrete wavelet transform and approximate entropy, Expert Systems with Applications, vol. 36, no. 2, pp. 2027-2036, Mar 2009.

[16] P. Stoica, R. Moses, Spectral Analysis of Signals. New Jersey: Prentice Hall 2005, ch. 2.

[17] X. Chen, I. Solomon, and K. Chon, Comparison of the Use of Approximate Entropy and Sample Entropy: Applications to Neural Respiratory Signal, in 2005 IEEE Engineering in Medicine and Biology 27th Annual Conference, vol. 4. IEEE, 2005, pp. 4212-4215.

[18] C. Bandt and B. Pompe, Permutation Entropy: A Natural Complexity Measure for Time Series, Physical Review Letters, vol. 88, no. 17, pp.174102, Apr 2002.

[19] U. R. Acharya, H. Fujita, V. K. Sudarshan, S. Bhat, and J. E. Koh, Application of entropies for automated diagnosis of epilepsy using EEG signals: A review, Knowledge-Based Systems, vol. 88, pp. 85-96, 2015.

[20] T. Meng et al., A survey on machine learning for data fusion, Information Fusion, vol. 57, pp. 115-129, 2020.

[21] F. Forooghifar et al., A Self-Aware Epilepsy Monitoring System for Real-Time Epileptic Seizure Detection, ACM/Springer Mobile Netw Appl (MONET), pp. 1-14, 2019.

[22] L. Breiman, Random Forests, Machine Learning 45, pp. 5-32, 2001 doi:10.1023/A:1010933404324

[23] S. Jenssen, E. J. Gracely, M. R. Sperling, How long do most seizures last? A systematic comparison of seizures recorded in the epilepsy monitoring unit, Epilepsia, vol. 47, no. 9, pp. 1499-503, Sep 2006. 